\title{
Scalar Particles Tunneling Radiation in the Demianski-Newman Spacetime with Influences of Quantum Gravity
}

\author{
Zhonghua Li $(\mathbb{D}$ \\ Institute of Theoretical Physics, China West Normal University, Nanchong, Sichuan 637002, China \\ Correspondence should be addressed to Zhonghua Li; sclzh888@163.com
}

Received 17 June 2020; Accepted 21 July 2020; Published 23 September 2020

Guest Editor: Farook Rahaman

Copyright (C) 2020 Zhonghua Li. This is an open access article distributed under the Creative Commons Attribution License, which permits unrestricted use, distribution, and reproduction in any medium, provided the original work is properly cited. The publication of this article was funded by SCOAP S $^{3}$

In this paper, using Hamilton-Jacobi ansatz, we investigate scalar particle tunneling radiation in the Demianski-Newman spacetime. We get the effective temperature with influences of quantum gravity and compare this temperature with the original temperature of the Demianski-Newman black hole. We find that it is similar to the case of fermions; for scalar particles, the influence of quantum gravity will also slow down the increase of Hawking temperatures, which naturally leads to remnants left in the evaporation.

\section{Introduction}

Hawking proposed that there exists radiation in black holes. The study of the black hole radiation is one of the important directions of black hole physics. WKB approximation is usually used to calculate the tunneling rate of emitted particles [1].

$$
\Gamma \propto \exp \left[-\operatorname{Im} \oint p_{r} d r\right]=\exp \left[-\operatorname{Im}\left(\int p_{r}^{\text {out }} d r-\int p_{r}^{\text {in }} d r\right)\right]
$$

The closed path above goes across the horizon and comes back. Related discussion about the tunneling rate of emitted particles is discussed in detail in [2-4].

The null geodesic method can be used to calculate the imaginary part of emitted particle action. It was used in the work of Parikh and Wilczek [5]. In this method, the Painleve coordinate transformation should be performed. The main characteristics of these coordinates are that they are stationary and nonsingular around the horizon. So one can obtain the imaginary part by canonical momenta and Hamilton canonical equations. The other method which can be used to calculate the imaginary part of emitted particles' action is the Hamilton-Jacobi Ansatz [6] which was first proposed in $[7,8]$. In this method, the action of the system accords with the Hamilton-Jacobi equation. Taking into account the property of the spacetime, one can make a separation of variables on the action $I=-\omega t+W(r)+\Phi(\phi, z)$. Then, inserting the results into the Hamilton-Jacobi equation, one can acquire the imaginary part. Many researchers extend this work to the tunneling radiation of fermions. The standard Hawking temperatures of the spherically symmetric and charged black holes were discussed in [9]. Other work about fermions' tunneling radiation is discussed in [10-18]. Reference [19] discussed a correction to the tunneling probability by taking into account the back reaction effect. In Ref. [20], features regarding the variation of the Hawking temperature, entropy, and tunneling rate were revealed, including corrections due to noncommutativity and back reaction. References $[21,22]$ discussed the quantum tunneling beyond semiclassical approximation and trace anomaly by the Hamilton-Jacobi method. Reference [23] discussed the Hawking radiation due to photon and gravitino tunneling. Topics related to the entropy-area spectrum of a black hole were discussed in Ref. [24-26]. Other important advances are discussed in $[27-30]$. 
Taking into account the theory of quantum gravity, there exists a minimal observable length [31-35]. Then, there is the generalized uncertainty principle (GUP)

$$
\Delta x \Delta p \geq \frac{\hbar}{2}\left[1+\beta \Delta p^{2}\right]
$$

where $\beta=\beta_{0}\left(l_{p}^{2} / \hbar^{2}\right), \beta_{0}$ is a dimensionless parameter, and $l_{p}$ is the Planck length. The derivation of the GUP is based on the modified fundamental commutation relations. Kempf et al. first modified commutation relations [36] and got $\left[x_{i}, p_{j}\right]=i \hbar \delta_{i j}\left[1+\beta p^{2}\right]$, where $x_{i}$ and $p_{i}$ are operators of position and momentum defined by

$$
\begin{aligned}
& x_{i}=x_{0 i}, \\
& p_{i}=p_{0 i}\left(1+\beta p^{2}\right),
\end{aligned}
$$

and here, $x_{0 i}$ and $p_{0 i}$ satisfy the basic quantum mechanics commutation relations $\left[x_{0 i}, p_{0 j}\right]=i \hbar \delta_{i j}$.

With consideration of the GUP, the cosmological constant problem was discussed, and the finiteness of the constant was derived in [37]. Based on the new form of GUP, the Unruh effect has been discussed in [38]. The quantum dynamics of the Friedmann-Robertson-Walker universe was derived in [39]. Some predictions on postinflation preheating in cosmology were made in [40]. Based on the generalization, the thermodynamics of the black holes were discussed again in [41-43] .

When effects of quantum gravity are taken into account, we investigated fermions' tunneling from the charged and rotating black strings [44]. We found that quantum gravity corrections slow down the increases of the temperatures, which naturally leads to remnants left in the evaporation. In [45], we discussed the tunneling of fermions when effects of quantum gravity are taken into account. We investigated two cases, black string and Kerr AdS black hole. We found that for black string, the uncharged and unrotating case, the correction of Hawking temperature is only affected by the mass of emitted fermions and the quantum gravitational corrections slow down the increases of the temperature, which naturally leads to remnants left in the evaporation, too. For another case, the Kerr AdS black hole, we found that the quantum gravitational corrections are not only determined by the mass of the emitted fermions but also affected by the rotating properties of the AdS black hole. So with consideration of the quantum gravity corrections, an offset around the standard temperature always exists.

In this paper, we do not discuss fermions but focus on scalar particle tunneling radiation in the DemianskiNewman spacetime with influences of quantum gravity. The Demianski-Newman black hole is similar to KerrNewman black hole. It is an extension of the Kerr-Newman black hole [46]. Its expression of metric is more complex than the Kerr-Newman black hole, so the calculation of rate of tunneling radiation is not easy relatively. Using the Hamilton-Jacobi ansatz, we investigate scalar particle tunnel- ing radiation in the Demianski-Newman spacetime. With consideration of quantum gravity, we get the effective temperature and compare this temperature with the original temperature of the Demianski-Newman black hole. We find that it is similar to the case of fermions; for scalar particles, the quantum gravity effects also slow down the increase of Hawking temperatures.

The rest is organized as follows. In the next section, based on the modified commutation relations of operators of position and momentum defined in [36], we will derive the generalized Klein-Gordon equation in curved spacetime. In Section 3, we investigate scalar particle tunneling radiation in the Demianski-Newman black hole. Section 4 is devoted to our conclusion.

\section{Generalized Klein-Gordon Equation in Curved Spacetime}

In this section, to account for the effects from quantum gravity, we first write the minimal length which is generalized uncertainty principle as

$$
\Delta x \Delta p \geq \frac{\hbar}{2}\left[1+\beta \Delta p^{2}\right]
$$

then we investigate the Klein-Gordon equation in curved spacetime with the effects of GUP. We adopt the position representation and take the effect of quantum gravity into consideration; the momentum operators are written as

$$
\begin{aligned}
x_{i} & =x_{0 i}, \\
p_{0 i} & =i \hbar \partial_{i}, \\
p_{i} & =p_{0 i}\left(1+\beta p^{2}\right) .
\end{aligned}
$$

Because $\beta$ is a small quantity, so we neglect the high orders of $\beta$ and get the square of momentum operators

$$
\begin{aligned}
p^{2}= & p_{i} p^{i}=-\hbar^{2}\left[1-\beta \hbar^{2}\left(\partial_{j} \partial^{j}\right)\right] \partial_{i} \cdot\left[1-\beta \hbar^{2}\left(\partial^{j} \partial_{j}\right)\right] \partial^{i} \\
& \simeq-\hbar^{2}\left[\partial_{i} \partial^{i}-2 \beta \hbar^{2}\left(\partial^{j} \partial_{j}\right)\left(\partial^{i} \partial_{i}\right)\right] .
\end{aligned}
$$

To take into account effects of quantum gravity, generalize the frequency as

$$
\tilde{\omega}=E\left(1-\beta E^{2}\right)
$$

where the energy operator takes the form of $E=i \hbar \partial_{0}$. According to the energy-mass shell condition: $p^{2}+m^{2}=E^{2}$, the modified expression of energy can be written as

$$
\tilde{E}=E\left[1-\beta\left(p^{2}+m^{2}\right)\right] .
$$

So we get the expression of the Klein-Gordon equation in the curved spacetime

$$
g^{\mu \nu} p_{\mu} p_{\nu} \Psi=-m^{2} \Psi
$$


To take into account the effects of quantum gravity, the above equation takes the form

$$
-(i \hbar)^{2} \partial^{t} \partial_{t} \Psi=\left[(i \hbar)^{2} \partial^{i} \partial_{i}+m^{2}\right] \Psi
$$

According to the above expression and keeping the foremost order of $\beta$, the generalized Klein-Gordon equation is rewritten as

$$
-(i \hbar)^{2} \partial^{t} \partial_{t} \Psi=\left[(i \hbar)^{2} \partial^{i} \partial_{i}+m^{2}\right]\left[1-2 \beta\left((i \hbar)^{2} \partial^{i} \partial_{i}+m^{2}\right)\right] \Psi .
$$

\section{Scalar Particle Tunneling Radiation in the Demianski-Newman Black Hole}

In this section, we research the tunneling radiation of scalar particles in the Demianski-Newman black hole by using the above generalized Klein-Gordon equation. The metric is given by [46]

$$
\begin{aligned}
d s^{2}= & -\frac{\Delta-a^{2} \sin ^{2} \theta}{\Sigma} d t^{2}+\frac{\Sigma}{\Delta} d r^{2}+\Sigma d \Theta^{2} \\
& -\frac{2\left(a B \sin ^{2} \theta-A \Delta\right)}{\Sigma} d t d \varphi+\frac{B^{2} \sin ^{2} \theta-A \Delta}{\Sigma} d \varphi^{2},
\end{aligned}
$$

where

$$
\begin{aligned}
& \Delta=r^{2}-2 M r+a^{2}-l^{2}, \\
& B=r^{2}+a^{2}+l^{2}, \\
& A=a \sin ^{2} \Theta+2 l \cos \theta, \\
& \Sigma=r^{2}+a^{2} \cos ^{2} \theta+l^{2}-2 a l \cos \theta .
\end{aligned}
$$

Here, $a$ reprents the angular momentum per unit mass and $M$ represents the black hole mass. Parameter $l$ in Demianski-Newman metric is an extended parameter relative to the Kerr-Newman black hole (when $l=0$, the Demianski-Newman black hole reduces to the KerrNewman black hole), it is related to rotation effect, and it can generate potential field. Reference [46] inferred that it may be some charge concerning spin. Black holes have inner and outer horizon; we only consider the outer horizon: $r_{h}^{+}$ $=M+\sqrt{M^{2}-a^{2}+l^{2}}$. The entropy of the black hole is defined as $S=\pi\left(r_{h^{+}}^{2}+a^{2}+l^{2}\right)$. Because the black hole rotates, it is not easy to research the particle tunneling directly, so we take the above metric dragging transformation as follows:

$$
\phi=\varphi-\Omega t
$$

where

$$
\Omega=\frac{a B \sin ^{2} \theta-A \Delta}{B^{2} \sin ^{2} \theta-A^{2} \Delta}
$$

Then, we get

$$
\begin{aligned}
d s^{2}= & -\frac{\Delta \Sigma}{\left(r^{2}+a^{2}+l^{2}\right)^{2}-\Delta A / \sin ^{2} \theta} d t^{2}+\frac{\Sigma}{\Delta} d r^{2} \\
& +\sum d \theta^{2}-+\frac{\left(r^{2}+a^{2}+l^{2}\right)^{2}-\left(a \sin ^{2} \theta+2 l \cos \theta\right)^{2} \Delta}{\Sigma} d \phi^{2} .
\end{aligned}
$$

To solve the generalized Klein-Gordon equation, we suppose that the wave function of the scalar particle takes the form

$$
\Psi=\exp \left[\frac{i}{\hbar} I(t, r, \theta, \phi)\right]
$$

In the wave function, $I$ represents the action of the scalar particle. Inserting the wave function into the generalized Klein-Gordon equation and the metric in DemianskiNewman black hole, considering the WKB approximation, keeping the leading order of $\beta$ and $\hbar$, and neglecting the higher orders of them, we get

$$
\begin{aligned}
\frac{1}{F}\left(\partial_{r} I\right)^{2}= & {\left[\left(G\left(\partial_{r} I\right)^{2}+\frac{1}{K^{2}}\left(\partial_{\theta} I\right)^{2}+\frac{1}{H^{2}}\left(\partial_{\phi} I\right)^{2}\right)+m^{2}\right] } \\
& \cdot\left(1-2 \beta\left[\left(G\left(\partial_{r} I\right)^{2}+\frac{1}{K^{2}}\left(\partial_{\theta} I\right)^{2}+\frac{1}{H^{2}}\left(\partial_{\phi} I\right)^{2}\right)+m^{2}\right]\right), \\
H^{2}= & \frac{\left(r^{2}+a^{2}+l^{2}\right)^{2}-\left(a \sin ^{2} \theta+2 l \cos \theta\right)^{2} \Delta}{\Sigma}, \\
K^{2}= & \Sigma .
\end{aligned}
$$

To get the solution of the above equation directly is not easy. In order to take separation of variables easily, we consider the form of the action of the scalar particle as follows:

$$
I=-(\omega-j \Omega) t+W(r)+j(\theta, \phi)
$$

where $\omega$ represents the energy of the emitted particle. We insert the above expression of action $I$ into the generalized Klein-Gordon equation by using separation of variables; then, we get

$$
C_{4}\left(\partial_{r} W\right)^{4}+C_{2}\left(\partial_{r} W\right)^{2}+C_{0}=0
$$

where

$$
\begin{aligned}
& C_{4}=-2 \beta G^{2}, \\
& C_{2}=G\left(1-4 \beta m^{2}\right), \\
& C_{0}=m^{2}-2 \beta m^{4}-\frac{(\omega-j \Omega)^{2}}{F} .
\end{aligned}
$$


Then, we can get the action of radial direction as follows:

$$
\begin{aligned}
W_{ \pm} & = \pm \int \frac{d r}{\sqrt{G F}} \sqrt{(\omega-j \Omega)^{2}-m^{2} F}\left(1+\beta m^{2}+\beta \frac{(\omega-j \Omega)^{2}}{F}\right) \\
& = \pm i \pi\left(\omega-j \Omega_{h^{+}}\right) \frac{r_{h^{+}}^{2}+a^{2}+l^{2}}{r_{h^{+}}-r_{h^{-}}}(1+\beta \xi)+(\text { real part }) .
\end{aligned}
$$

In the above equation, +/- represents outward/interior solution, respectively, where

$$
\begin{gathered}
\Omega_{h^{+}}=\frac{a}{r_{h^{+}}^{2}+a^{2}+l^{2}}, \\
r_{h^{+}}=M+\sqrt{M^{2}-a^{2}+l^{2}}, \\
F=\frac{\Delta \Sigma}{\left(r^{2}+a^{2}+l^{2}\right)^{2}-\Delta A^{2} / \sin ^{2} \theta}, \\
G=\frac{\Delta}{\Sigma} .
\end{gathered}
$$

The expression of $\xi$ is pretty complicated, so we do not write it here. Then, with WKB approximation, the tunneling rate of the scalar particle can be written as

$$
\begin{aligned}
\Gamma & =\frac{P_{\text {out }}}{P_{\text {in }}}=\frac{\exp \left(-2 I_{m} I_{+}\right)}{\exp \left(-2 I_{m} I_{-}\right)}=\exp \left[-2\left(I_{m} I_{+}-I_{m} I_{-}\right)\right] \\
& =\exp \left[4 \pi\left(\omega-j \Omega_{h^{+}}\right) \frac{r_{h^{+}}^{2}+a^{2}+l^{2}}{r_{h^{+}}-r_{h^{-}}}(1+\beta \xi)\right] .
\end{aligned}
$$

According to the expression of the Boltzmann factor, we can get the corrected Demianski-Newman temperature as

$$
T=\frac{r_{h^{+}}-r_{h^{-}}}{4 \pi\left(r_{h^{+}}^{2}+a^{2}+l^{2}\right)(1+\beta \xi)}=\frac{T_{0}}{1+\beta \xi}=T_{0}(1-\beta \xi),
$$

where the original temperature of the Demianski-Newman black hole is

$$
T_{0}=\frac{r_{h^{+}}-r_{h^{-}}}{4 \pi r_{h^{+}}^{2}+a^{2}+l^{2}}
$$

\section{Discussion and Conclusions}

In this paper, we discuss scalar particle tunneling radiation in the Demianski-Newman spacetime with the effects of quantum gravity. According to the effective Demianski-Newman temperature, apparently, we can conclude that the corrected Demianski-Newman temperature is lower than the original temperature of the Demianski-Newman black hole. Because $\beta$ is a small quantity, it is similar to the case of fermions; for scalar particles, the influence of quantum gravity will also slow down the evaporation of the Demianski-Newman black hole, so when the black hole is at the balance point, there are remnants in the black hole.

\section{Data Availability}

All data used to support the findings of this study are included within the article.

\section{Conflicts of Interest}

The authors declare that they have no conflicts of interest.

\section{Acknowledgments}

This work is supported by the Fundamental Research Funds of China West Normal University (13C009). We thank L. M. Zhang for her participation in the early stage of this work.

\section{References}

[1] V. Akhmedova, T. Pilling, A. de Gill, and D. Singleton, "Comments on anomaly versus $\mathrm{WKB} /$ tunneling methods for calculating Unruh radiation," Physics Letters B, vol. 673, no. 3, pp. 227-231, 2009.

[2] E. T. Akhmedov, V. Akhmedova, T. Pilling, and D. Singleton, "Thermal radiation of various gravitational backgrounds," International Journal of Modern Physics A, vol. 22, no. 8-9, pp. 1705-1715, 2012.

[3] B. D. Chowdhury, "Problems with tunneling of thin shells from black holes," Pramana, vol. 70, no. 1, pp. 3-26, 2008.

[4] E. T. Akhmedov, V. Akhmedova, and D. Singleton, "Hawking temperature in the tunneling picture," Physics Letters B, vol. 642, no. 1-2, pp. 124-128, 2006.

[5] M. K. Parikh and F. Wilczek, "Hawking radiation as tunneling," Physical Review Letters, vol. 85, no. 24, pp. 5042-5045, 2000.

[6] M. Angheben, M. Nadalini, L. Vanzo, and S. Zerbini, "Hawking radiation as tunneling for extremal and rotating black holes," Journal of High Energy Physics, vol. 2005, no. 5, p. 14, 2005.

[7] K. Srinivasan and T. Padmanabhan, "Particle production and complex path analysis," Physical Review D, vol. 60, no. 2, article $024007,1999$.

[8] S. Shankaranarayanan, T. Padmanabhan, and K. Srinivasan, "Hawking radiation in different coordinate settings: complex paths approach," Classical and Quantum Gravity, vol. 19, no. 10, pp. 2671-2687, 2002.

[9] R. Kerner and R. B. Mann, "Fermions tunnelling from black holes," Classical and Quantum Gravity, vol. 25, no. 9, article 095014, 2008.

[10] R. Kerner and R. B. Mann, "Charged fermions tunnelling from Kerr-Newman black holes," Physics Letters B, vol. 665, no. 4, pp. 277-283, 2008.

[11] J. Ahmed and K. Saifullah, "Hawking temperature of rotating charged black strings from tunneling," Journal of Cosmology and Astroparticle Physics, vol. 2011, no. 11, article 23, 2011.

[12] J. Ahmed and K. Saifullah, "Hawking radiation of Dirac particles from black strings," Journal of Cosmology and Astroparticle Physics, vol. 2011, no. 8, article 11, 2011.

[13] R. Li and J.-R. Ren, "Dirac particles tunneling from BTZ black hole," Physics Letters B, vol. 661, no. 5, pp. 370-372, 2008.

[14] D.-Y. Chen, Q.-Q. Jiang, and X.-T. Zu, "Hawking radiation of Dirac particles via tunnelling from rotating black holes in de 
Sitter spaces," Physics Letters B, vol. 665, no. 2-3, pp. 106-110, 2008.

[15] Q.-Q. Jiang, “Dirac particle tunneling from black rings," Physical Review D, vol. 78, no. 4, article 044009, 2008.

[16] R. D. Criscienzo and L. Vanzo, "Fermion tunneling from dynamical horizons," Europhysics Letters, vol. 82, no. 6, article 60001, 2008.

[17] S. A. Hayward, R. D. Criscienzo, M. Nadalini, L. Vanzo, and S. Zerbini, "Local hawking temperature for dynamical black holes," Classical and Quantum Gravity, vol. 26, no. 6, article 062001, 2009.

[18] B. R. Majhi, "Fermion tunneling beyond semiclassical approximation," Physical Review D, vol. 79, no. 4, article 044005, 2009.

[19] R. Banerjee and B. R. Majhi, "Quantum tunneling and back reaction," Physics Letters B, vol. 662, no. 1, pp. 62-65, 2008.

[20] R. Banerjee, B. R. Majhi, and S. Samanta, "Noncommutative black hole thermodynamics," Physical Review D, vol. 77, no. 12, article 124035, 2008.

[21] R. Banerjee and B. R. Majhi, "Quantum tunneling beyond semiclassical approximation," Journal of High Energy Physics, vol. 2008, no. 6, p. 095, 2008.

[22] R. Banerjee and B. R. Majhi, "Quantum tunneling and trace anomaly," Physics Letters B, vol. 674, no. 3, pp. 218-222, 2009.

[23] B. R. Majhi and S. Samanta, "Hawking radiation due to photon and gravitino tunneling," Annals of Physics, vol. 325, no. 11, pp. 2410-2424, 2010.

[24] R. Banerjee and B. R. Majhi, "Hawking black body spectrum from tunneling mechanism," Physics Letters B, vol. 675, no. 2, pp. 243-245, 2009.

[25] R. Banerjee, B. R. Majhi, and E. C. Vagenas, "Quantum tunneling and black hole spectroscopy," Physics Letters B, vol. 686, no. 4-5, pp. 279-282, 2010.

[26] B. R. Majhi, "Hawking radiation and black hole spectroscopy in Hořava-Lifshitz gravity," Physics Letters B, vol. 686, no. 1, pp. 49-54, 2010.

[27] R. Banerjee and B. R. Majhi, "Connecting anomaly and tunneling methods for the Hawking effect through chirality," Physical Review D, vol. 79, no. 6, article 064024, 2009.

[28] R. Banerjee, B. R. Majhi, and E. C. Vagenas, "A note on the lower bound of black-hole area change in the tunneling formalism," Europhysics Letters, vol. 92, no. 2, p. 20001, 2010.

[29] R. Banerjee, C. Kiefer, and B. R. Majhi, "Quantum gravitational correction to the Hawking temperature from the Lemaître-Tolman-Bondi model," Physical Review D, vol. 82, no. 4 , article $044013,2010$.

[30] K. Bhattacharya and B. R. Majhi, "Temperature and thermodynamic structure of Einstein's equations for a cosmological black hole," Physical Review D, vol. 94, no. 2, article 024033, 2016.

[31] P. K. Townsend, "Small-scale structure of spacetime as the origin of the gravitational constant," Physical Review D, vol. 15, no. 10, pp. 2795-2801, 1977.

[32] D. Amati, M. Ciafaloni, and G. Veneziano, "Can spacetime be probed below the string size?," Physics Letters B, vol. 216, no. 12, pp. 41-47, 1989.

[33] K. Konishi, G. Paffuti, and P. Provero, "Minimum physical length and the generalized uncertainty principle in string theory," Physics Letters B, vol. 234, no. 3, pp. 276-284, 1990.
[34] L. J. Garay, "Quantum gravity and minimum length," International Journal of Modern Physics A, vol. 10, no. 2, pp. 145-165, 2012.

[35] G. Amelino-Camelia, "Relativity in spacetimes with shortdistance structure governed by an observer-independent (Planckian) length scale," International Journal of Modern Physics D, vol. 11, no. 1, pp. 35-59, 2012.

[36] A. Kempf, G. Mangano, and R. B. Mann, "Hilbert space representation of the minimal length uncertainty relation," Physical Review D, vol. 52, no. 2, pp. 1108-1118, 1995.

[37] L. N. Chang, D. Minic, N. Okamura, and T. Takeuchi, "Effect of the minimal length uncertainty relation on the density of states and the cosmological constant problem," Physical Review D, vol. 65, no. 12, article 125028, 2002.

[38] B. R. Majhi and E. C. Vagenas, "Modified dispersion relation, photon's velocity, and Unruh effect," Physics Letters B, vol. 725, no. 4-5, pp. 477-480, 2013.

[39] M. V. Battisti and G. Montani, "The big-bang singularity in the framework of a generalized uncertainty principle," Physics Letters B, vol. 656, no. 1-3, pp. 96-101, 2007.

[40] W. Chemissany, S. Das, A. F. Ali, and E. C. Vagenas, "Effect of the generalized uncertainty principle on post-inflation preheating," Journal of Cosmology and Astroparticle Physics, vol. 2011, no. 12, article 17, 2011.

[41] W. Kim, E. J. Son, and M. Yoon, "Thermodynamics of a black hole based on a generalized uncertainty principle," Journal of High Energy Physics, vol. 2008, no. 1, article 35, 2008.

[42] L. Xiang and X. Q. Wen, "A heuristic analysis of black hole thermodynamics with generalized uncertainty principle," Journal of High Energy Physics, vol. 2009, no. 10, article 46, 2009.

[43] A. Bina, S. Jalalzadeh, and A. Moslehi, "Quantum black hole in the generalized uncertainty principle framework," Physical Review D, vol. 81, no. 2, article 023528, 2010.

[44] D. Chen and Z. Li, "Remarks on remnants by fermions tunnelling from black strings," Advances in High Energy Physics, vol. 2014, Article ID 620157, 9 pages, 2014.

[45] Z.-H. Li and L.-M. Zhang, "Fermions tunnelling from black string and Kerr AdS black hole with consideration of quantum gravity," International Journal of Theoretical Physics, vol. 54, no. 6, pp. 1739-2118, 2015.

[46] S.-W. Gao, Z. Peng, and Z. Zhao, "The thermal property of Demianski-Newman black hole and the generalized Bekenstein-Smarr formula," Journal of Beijing Normal University, vol. 37, no. 4, pp. 493-498, 2001. 